\title{
Freezing and melting of water in porous geomaterials studied by magnetic resonance
}

Dimitri Bytchenkoff, Stéphane Rodts, Pascal Moucheront, David Hautemayou, Cédric Mézière, Paméla Faure and Teddy FenChong

Université ParisEst U.R. Navier (UMR 113 CNRS); 2 allée Kepler, 77420 Champs sur Marne, France

We intend to study mechanical effects of freezing and thawing on bulky samples of concrete by nuclear resonance high resolution spectroscopy and imaging (MRI). Variously functionalised three dimensional images of liquid water in the porous media can be obtained by MRI. NMR spectroscopy, on the other hand, can be used to directly measure the total quantity of liquid water as a function of temperature. Moreover, measurements of magnetic spin relaxation rates and diffusion can provide information about distribution of pore sizes (see the figure below) and their connectivity as well as the flow of water through porous media. Knowledge of all these properties is required to fully understand behaviour of porous materials under freezing and thawing.

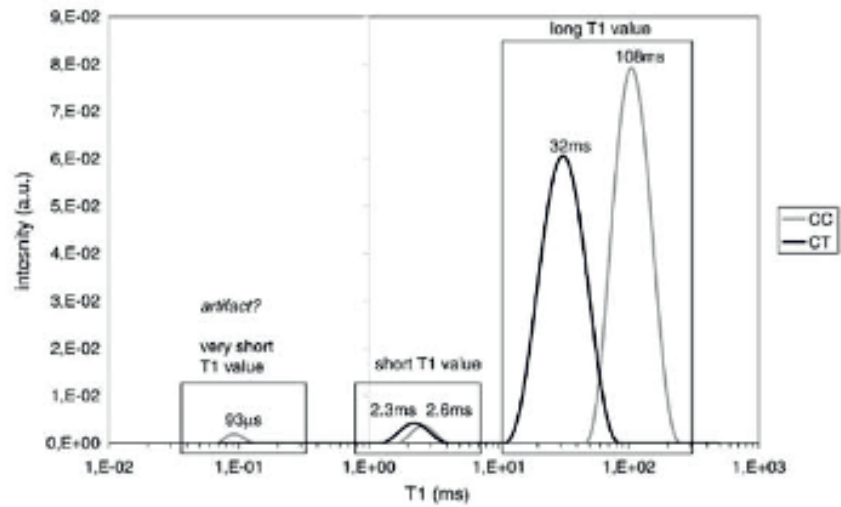

This is an Open Access article distributed under the terms of the Creative Commons Attribution-Noncommercial License 3.0, which permits unrestricted use, distribution, and reproduction in any noncommercial medium, provided the original work is properly cited. 
We have designed and built a cooler (see the figure below) that can be inserted into the probe of an NMR spectrometer to bring the temperature a balky cylindrical sample down to $100^{\circ} \mathrm{C}$. The present version of the cooler is intended first and foremost for experiments in which the NMR signal of water hydrogen will be registered. Therefore, the part of the device that will be placed in the active zone of the NMR probe is made of nonmagnetic nonconductive materials containing no hydrogen isotope to avoid that it emits any extra signal that could interfere with that emanating from water protons of the sample. Furthermore, a cryostat, pump, air compressor and several other auxiliary components of the device were all put outside the Faraday cage built around the NMR spectrometer to minimise noise that they can induce in the NMR probe. Optical fiber sensors were built into the cell to provide for monitoring temperature and mechanical deformations by the means other than NMR. The cooler was designed to use a commercial coolant 'Galden' for heat transfer and has advantage of maintaining the radio frequency (RF) field emitter/receiver coil of the spectrometer at room temperature.

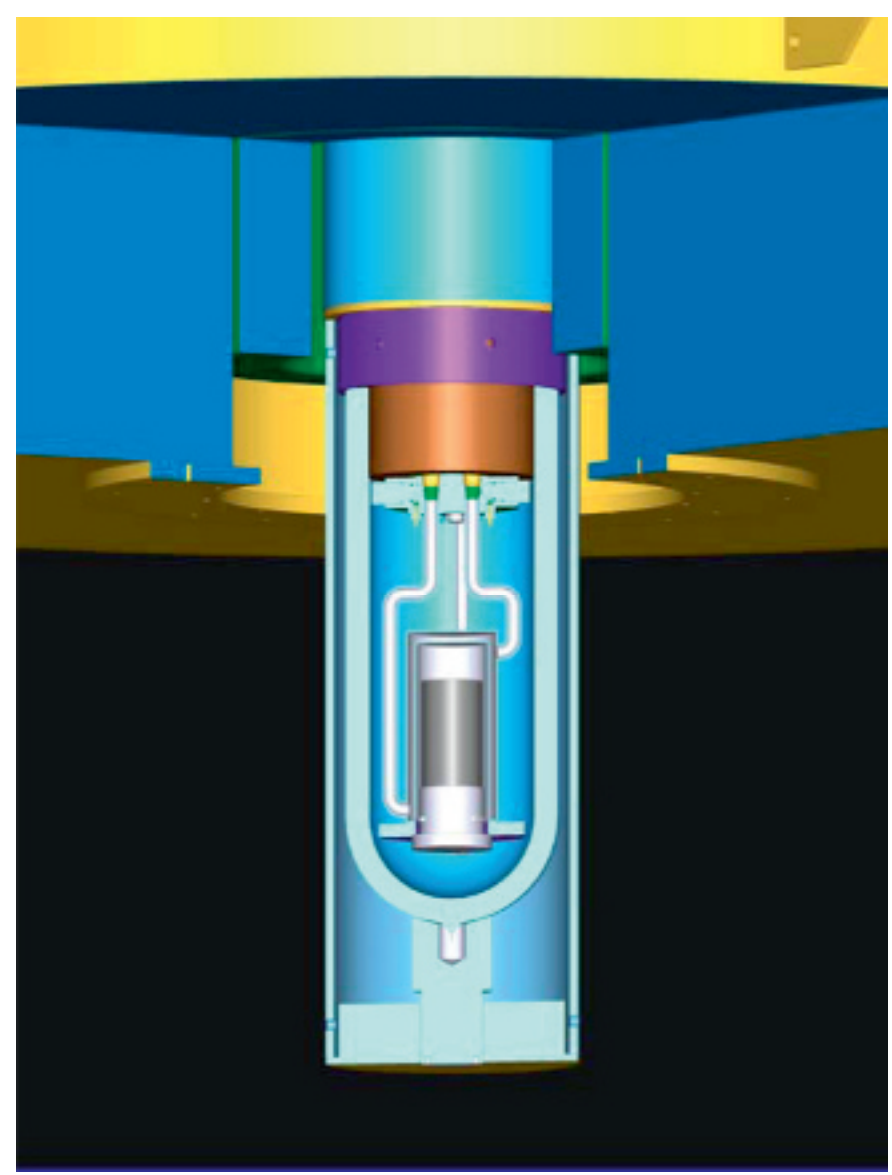

